\title{
Discontinuous Buckling of Wide Beams and Metabeams
}

\section{Citation}

Coulais, Corentin, Johannes T. B. Overvelde, Luuk A. Lubbers, Katia Bertoldi, and Martin van Hecke. 2015. "Discontinuous Buckling of Wide Beams and Metabeams." Physical Review Letters 115 (4) (July 21). doi:10.1103/physrevlett.115.044301.

\section{Published Version}

doi:10.1103/PhysRevLett.115.044301

\section{Permanent link}

http://nrs.harvard.edu/urn-3:HUL.InstRepos:27662697

\section{Terms of Use}

This article was downloaded from Harvard University's DASH repository, and is made available under the terms and conditions applicable to Other Posted Material, as set forth at http:// nrs.harvard.edu/urn-3:HUL.InstRepos:dash.current.terms-of-use\#LAA

\section{Share Your Story}

The Harvard community has made this article openly available.

Please share how this access benefits you. Submit a story.

\section{Accessibility}




\title{
Discontinuous Buckling of Wide Beams and Metabeams
}

\author{
Corentin Coulais, ${ }^{1,2}$ Johannes T. B. Overvelde, ${ }^{3}$ Luuk A. Lubbers, ${ }^{1,2}$ Katia Bertoldi, ${ }^{3}$ and Martin van Hecke ${ }^{1,2}$ \\ ${ }^{1}$ Huygens-Kamerlingh Onnes Lab, Universiteit Leiden, P.O. box 9504, 2300 RA Leiden, The Netherlands \\ ${ }^{2}$ FOM Institute AMOLF, Science Park 104, 1098 XG Amsterdam, The Netherlands \\ ${ }^{3}$ School of Engineering and Applied Sciences, Harvard University, Cambridge, Massachusetts 02138, USA
}

(Received 22 October 2014; published 21 July 2015)

\begin{abstract}
We uncover how nonlinearities dramatically alter the buckling of elastic beams. First, we show experimentally that sufficiently wide ordinary elastic beams and specifically designed metabeams-beams made from a mechanical metamaterial—exhibit discontinuous buckling, an unstable form of buckling where the postbuckling stiffness is negative. Then we use simulations to uncover the crucial role of nonlinearities, and show that beams made from increasingly nonlinear materials exhibit an increasingly negative postbuckling slope. Finally, we demonstrate that for sufficiently strong nonlinearity, we can observe discontinuous buckling for metabeams as slender as $1 \%$ numerically and $5 \%$ experimentally.
\end{abstract}

DOI: 10.1103/PhysRevLett.115.044301

Buckling of straight beams under sufficient load $F$ is perhaps the most basic example of an elastic instability. This instability can be captured in models of varying degree of sophistication [1], starting from Euler's elastica which describes the bending of elastic lines and is appropriate for slender beams [2]. Even the simplest analysis gives an excellent estimate of the critical buckling load $F_{c}$, crucial for engineering [3]. The relation between $F$ and compressive displacement $u$ for a beam of length $L$ takes the form $\left(F-F_{c}\right) / F_{c}=S u / L$, with the elastica predicting that the postbuckling slope $S$ equals $1 / 2$, independent of boundary conditions-see Fig. 1(a).

Here we describe how nonlinearities-due to large strains in wide beams or due to strong nonlinearities in metabeams-dramatically alter this postbuckling scenario. In particular we find that sufficiently strong nonlinearities lead to discontinuous buckling, a novel form of buckling where the force in the postbuckling regime decreases for increasing deformation, so that $S<0$.

First, we perform experiments on ordinary elastic beams and show that they undergo discontinuous buckling when the beams' aspect ratio exceeds 12\%-see Fig. 1(b). Second, we create beams out of a strongly nonlinear mechanical metamaterial, and show that such beams undergo discontinuous buckling when the material's nonlinearity becomes sufficiently strong, even for slender beams-see Fig. 1(c). Third, using finite element simulations, we uncover a significant nonlinear contribution to the total elastic energy of both wide beams and metabeams, which we suggest pushes the beam away from the Euler limit and causes discontinuous buckling. To test this hypothesis, we numerically study a range of metabeams and show that the strength of the nonlinearity of their stressstrain relation and their postbuckling slopes are strongly correlated. Finally, we present numerical evidence that a judicious choice of metamaterial parameters can cause
PACS numbers: 46.70.De, 62.20.mq, 81.05.Xj, 81.05.Zx

arbitrarily slender beams to exhibit discontinuous buckling, and experimentally achieve discontinuous buckling for metabeams as slender as 5\%. Our work illuminates the crucial role of nonlinearities for buckling, and paves the way for novel strategies where mechanical metamaterials are used to qualitatively change and control the nature of elastic instabilities.

Discontinuous buckling.-We first perform experiments on the buckling of both ordinary elastic beams and metabeams. To minimize gravitational effects we perform density-matched experiments in a bath of water. We rigidly mount the beams (using silicon glue) to the top and bottom plate of an Instron 5965 uniaxial testing device equipped with a $100 \mathrm{~N}$ load cell, allowing us to measure the axial force $F$ (accuracy $10^{-4} \mathrm{~N}$ ) as a function of the axial compressive displacement $u$ (accuracy $10^{-3} \mathrm{~mm}$ ).
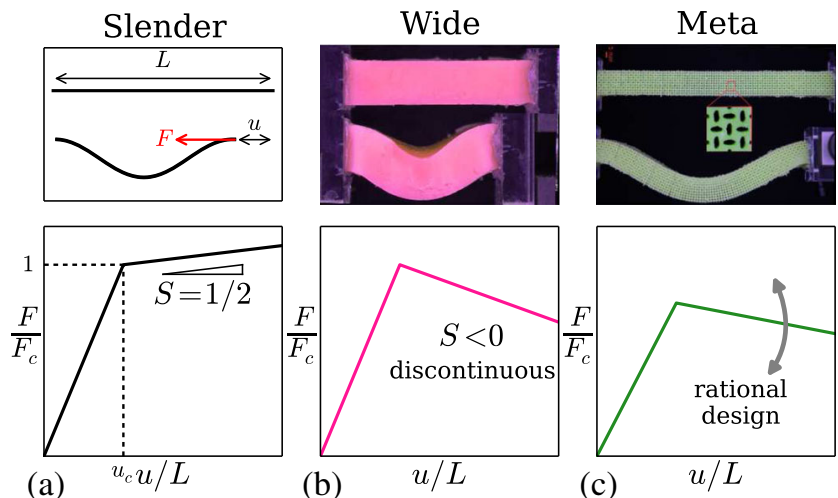

(b)

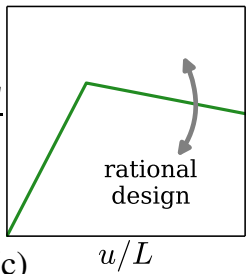

FIG. 1 (color online). Buckling of slender beams, wide beams, and metabeams. (a) Slender beams in their undeformed (top) and buckled (bottom) states. The force displacement curve for slender beams has a postbuckling slope $S=1 / 2$. (b) Discontinuous $(S<0)$ buckling of wide beams. (c) Slender metabeams consisting of a nonlinear elastic metamaterial can also exhibit discontinuous buckling. 

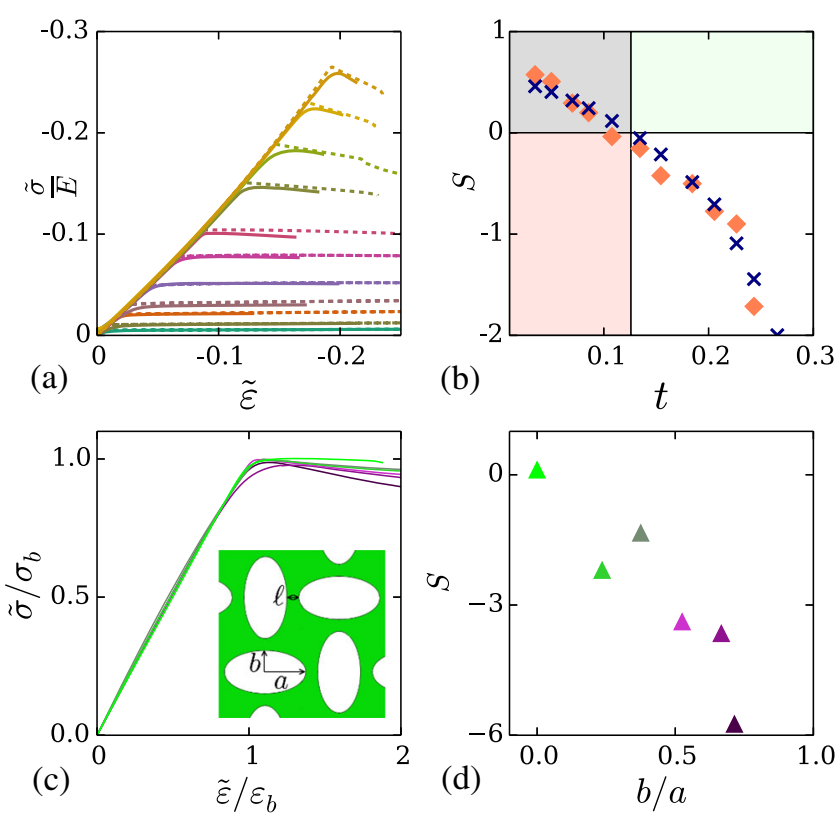

FIG. 2 (color online). Discontinuous buckling. (a) Forcedisplacement curves for beams with aspect ratios ranging from $t=0.034$ (lowest curve) to $t=0.284$ (highest curve) for experiments (solid lines) and finite elements simulations (dashed lines). Here, $\tilde{\sigma} / E:=-F /(E w d)$ and $\tilde{\varepsilon}:=-u / L$. (b) The postbuckling slope in experiments (orange diamonds) and simulations (blue crosses), $S$, increasingly deviates from the elastica limit $1 / 2$ for large $t$. Discontinuous buckling $(S<0)$ occurs for $t>0.12$. (c) Force-displacement curves (rescaled by the buckling stress and strain, $\sigma_{b}$ and $\varepsilon_{b}$ ) for metabeams of $t=0.10, \ell=0.3$, and various values of $b / a$. Here $\sigma_{b}$ and $\varepsilon_{b}$ denote the values at buckling. Inset: geometry of our metamaterial. (d) Corresponding postbuckling slope $S$ vs $b / a$.

The wide beams are solid, rectangular beams of length $L=45 \mathrm{~mm}$, depth $d=35 \mathrm{~mm}$, and widths ranging from $w=1.55 \mathrm{~mm}$ to $w=12.85 \mathrm{~mm}$ (aspect ratios, or thicknesses, $t:=w / L$ up to 0.27 ) [4]. These are created by molding a well-characterized silicon rubber [5]. The metabeams consist of a rubber mechanical metamaterial designed to allow tuning of the effective stress-strain nonlinearity. We take inspiration from a recently proposed mechanical metamaterial, which consists of a $2 \mathrm{D}$ elastic slab patterned by a regular array of circular holes. Such a system exhibits an elastic instability under compression leading to a transformation to a pattern of mutually orthogonal ellipses and a sharp kink in the stress-strain relation [6-9]. Here we use instead metamaterials with elliptical holes [Fig. 2(c)], which break rotational symmetry and suppress this elastic instability $[10,11]$, transforming the sharp kink into a controllable nonlinearity of the stress-strain relation. We have created six metabeams $(L=220 \mathrm{~mm}, d=29 \mathrm{~mm}, w=24 \mathrm{~mm}$, $t=0.10$, and $E=1.1 \times 10^{6} \mathrm{~Pa}$ ) with a varying aspect ratio between the ellipses by $3 \mathrm{D}$ printing molds in which we cast a silicon rubber [12] . Each beam contains $9 \times 98=882$ holes, where the strongest nonlinearities occur for nearcircular holes.

We plot the experimental force-displacement curves and the postbuckling slope $S$ for wide beams in Figs. 2(a) and 2(b), and for metabeams in Figs. 2(c) and 2(d). For all beams, there is an initially near-linear elastic behavior with a sudden departure from linearity as a result of buckling [13]. Our first main result is that for ordinary beams with $t \gtrsim 0.12$, or for metabeams with near-circular holes, the postbuckling slope becomes negative. Hence, under increasing load, such beams exhibit discontinuous buckling. Note that a negative stiffness is readily observed in other mechanical systems such as buckling shells [3], the Roorda frame, and pipes [18], where it is associated with asymmetric or saddle node bifurcations. Together with wrinkling membranes [19-21], this example is one of the few where a negative stiffness is reported for a mechanical system undergoing a symmetry breaking pitchfork bifurcation.

Numerical approach.-In order to understand discontinuous buckling, we make extensive use of finite element simulations and conduct a fully nonlinear analysis within the commercial package ABAQUS/STANDARD. To calibrate the constitutive relation, we first focus on wide, ordinary beams. These undergo substantial uniaxial compression before buckling, pushing the physics beyond that of simple linear elasticity. Such rubberlike materials are well described by the incompressible neo-Hookean formulation of elasticity, which leads to a nonlinear stress-strain relation [22,23]. In Fig. 2(a) we compare our experimental data to finite element simulations of such a neo-Hookean 3D model, with realistic (fixed) boundary conditions, for $E=250 \mathrm{kPa}$ and $\nu=0.49999$ [24], and find excellent agreement between experiments and simulations, validating the use of this weakly nonlinear model. In the remainder of the Letter we will use 2D (plain strain) simulations [25]: (i) of the full metabeam to extract $S$; (ii) of a unit cell with periodic boundary conditions to determine the effective stress-strain relation for a uniaxial test.

Nonlinearity.-We now illustrate and quantify the role of nonlinearity for the stresses and elastic energies in the postbuckling regime, comparing three beams: a slender ordinary beam close to the Euler limit, a wide beam, and a metabeam. In Figs. 3(a)-3(c) we show the effective stressstrain relation of these beams (extracted from our numerical simulations), as well as the range of axial strains and stresses throughout the whole 3D slender and wide beams, and throughout the whole 2D metabeam. To facilitate comparison of the strength of the nonlinearities, all data are taken at $\tilde{\varepsilon} / \varepsilon_{b}=120 \%$, where $\varepsilon_{b}$ denotes the onset of buckling. Figures 3(a) and 3(b) illustrate that while for a slender beam $(t=0.034)$ the strains only span a limited range $\left(\Delta \tilde{\varepsilon}=1.2 \times 10^{-3}\right)$ so that the stresses are not very sensitive to the neo-Hookean nonlinearity, for a wide beam $(t=0.134)$ the strains span a larger range $\left(\Delta \tilde{\varepsilon}=1.8 \times 10^{-1}\right)$ and the stresses thus deviate 

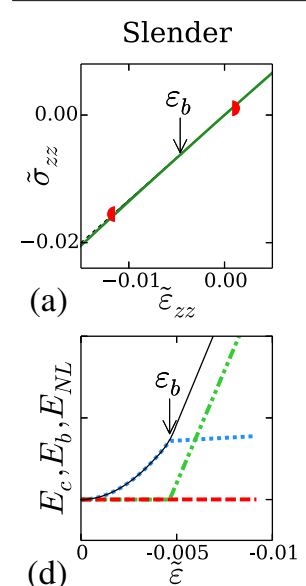

(b)
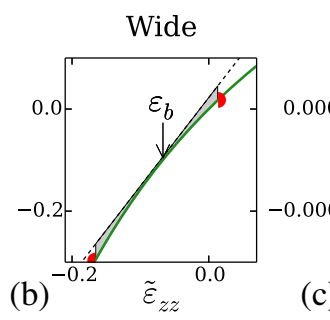

Meta

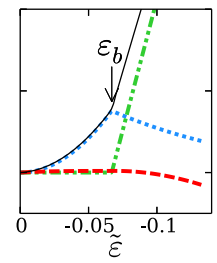

(f)
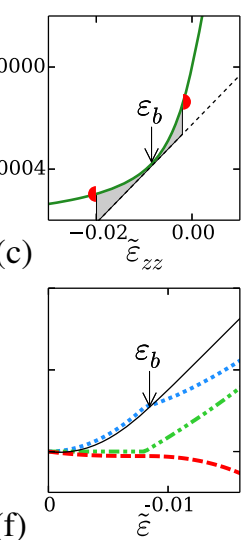

FIG. 3 (color online). (a)-(c) Stresses in 3D slender (a) $L \times d=$ $45 \mathrm{~mm} \times 35 \mathrm{~mm}, t=0.034$ ) and wide (b) same $L \times d, t=0.134$ ) beams and for a 2D metabeam [(c) $t=0.054]$. The green curves depict the stress-strain relation, the black dashed lines denote linear fits around $\tilde{\varepsilon_{b}}$, and the red semicircles denote the range of stresses and strains throughout the beams for $\tilde{\varepsilon} / \tilde{\varepsilon_{b}}=1.2$. (d)-(f) Energies $E_{c}$ (dotted, blue line), $E_{b}$ (dot-dashed, green line), and $E_{N L}$ (dashed, red line) [Eqs. (3)-(5)] and total energy $E_{t}$ (black) vs. displacement $\tilde{\varepsilon}$. A quadratic fit to $E_{t}$ in the postbuckling regime gives $\partial^{2} E_{t} / \partial \tilde{\varepsilon}^{2}=1.6 \times 10^{-2}$ (a), $-1.1 \times 10^{-1}(\mathrm{~b})$, and $-4.4 \times 10^{-2}(\mathrm{c})$.

significantly from the linear (Euler) case. Moreover, Fig. 3(c) illustrates that metabeams with a strongly nonlinear stress-strain relation exhibit stresses that deviate significantly from the linear case even for small strains. Clearly both the width (setting the range of strains) and the nonlinearity of the material (setting the curvature of the stress-strain relation) play a role in determining the deviations from the Euler limit.

To quantify the role of nonlinearity, we will now determine the contributions to the elastic energy of bending, compression, and nonlinearity in the regime close to the buckling strain $\varepsilon_{b}$. To do so, we need to determine the constitutive law as well as an equation for the axial strain as a function of $x$, the horizontal coordinate across the beam width $w$. For the constitutive law we expand the stressstrain relation to quadratic order around $\varepsilon_{b}$ :

$$
\tilde{\sigma}_{z z}=E\left(\tilde{\varepsilon}_{z z}+\eta\left(\tilde{\varepsilon}_{z z}-\varepsilon_{b}\right)^{2}\right),
$$

where $\eta$ quantifies the nonlinearity [26]. The axial strain profile is expanded as

$$
\tilde{\varepsilon}_{z z}=\varepsilon+\kappa x,
$$

where $\kappa$ and $\varepsilon$ are, respectively, the curvature and the compression of the neutral plane of the beam. Neglecting shear (which can be shown to be subdominant [27]), the elastic energy can then be determined as $E_{t}=$ $\int d V \int \tilde{\sigma}_{z z} d \tilde{\varepsilon}_{z z}=E_{c}+E_{b}+E_{N L}$, with

$$
\begin{gathered}
E_{b}=\frac{E d w^{3}}{12} \int_{0}^{L} d s \kappa^{2}, \\
E_{c}=E d w \int_{0}^{L} d s \varepsilon^{2}, \\
E_{N L}=\eta \frac{E d w}{3} \int_{0}^{L} d s\left(\frac{w^{2}}{4}\left(\varepsilon-\varepsilon_{b}\right) \kappa^{2}+\left(\varepsilon-\varepsilon_{b}\right)^{3}+\varepsilon_{b}^{3}\right),
\end{gathered}
$$

where $s$ is the curvilinear coordinate of the beam. We note that the elastica only uses $E_{b}$, whereas extensible elastica uses both $E_{b}$ and $E_{c}$, but does not take nonlinearities, such as those encountered in neo-Hookean materials, into account [22,23]—consequently, the postbuckling slope in such models remains positive up to unrealistically large aspect ratios [28,29]. We have recently developed a full theoretical description taking $E_{N L}$ into account, which is quantitatively consistent with our experimental and numerical data, and which will appear separately [27].

Here we focus on comparing the contributions of these energies for slender beams, wide beams, and metabeams, and extract $\kappa$ and $\varepsilon$ from the neutral plane or line of the simulated beams [Figs. 3(d)-3(f)]. For the slender beam, the nonlinear term remains small, and after buckling, $E_{t}$ grows faster than linear so that $S:=-\left(E F_{c}\right)^{-1} \partial \tilde{\sigma} / \partial \tilde{\varepsilon}=$ $\left(1 / F_{c}\right) \partial^{2} E_{t} / \partial \tilde{\varepsilon}^{2}$ is positive [Fig. 3(d)], as expected. In contrast, for the wide beam, the nonlinear contribution becomes significant and induces a sublinear increase of the total energy, leading to $S<0$ [Fig. 3(e)]. For metabeams, the nonlinear contribution becomes similarly important and leads to $S<0$ also.

We suggest that the significant nonlinear contribution upsets the energy balance and perturbs the beam away from the slender beam limit. Figures 3(d)-3(f) illustrate the opposite nature of the changes in compressive energy between wide beams $(\eta<0)$ and metabeams $(\eta>0)$. Wide beams lower their energy by extending after buckling (in contrast to slender beams), due to the neo-Hookean nonlinearity which is stiffening under compression; metabeams lower their energy by shortening more than slender beams after buckling, due to the constitutive nonlinearity which is softening under compression. In both cases, stronger nonlinearities lead to an increasing deviation from the Euler limit, leading to a change in the beam geometry and eventually to discontinuous buckling.

Tunable nonlinearity in metabeams. - To establish the connection between the nonlinearity of the metamaterial and the postbuckling slope of the metabeams, we perform extensive simulations of our 2D homogeneous metamaterials and metabeams, scanning the metaparameters $\ell$ and $e:=1-b / a$ as well as beam thickness $t$. Figure 4 compares $\eta$ and $S$ for a range of $\ell$ and $e:=1-b / a$, for beams of $t=0.054$. Clearly a smaller gap between the holes $\ell$ leads to larger nonlinearities, whereas the trend with $e$ is 

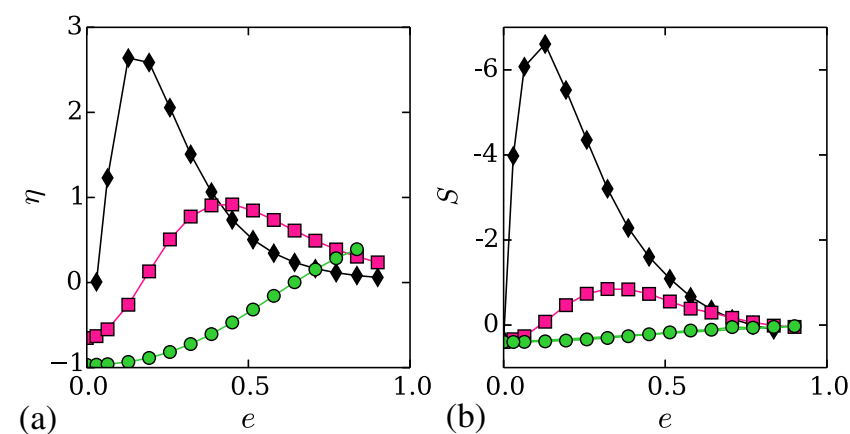

FIG. 4 (color online). Numerical simulations of 2D metabeams of $5.4 \%$ aspect ratio $\left(\varepsilon_{b}=-0.0096\right)$ and $6 \times 111$ holes for the nonlinearity $\eta$ (a) and postbuckling slope $S$ (b) vs $e$ for $\ell=0.13$ (black diamonds), $\ell=0.23$ (pink squares), and $\ell=0.42$ (green circles). See Ref. [30] for movies.

nonmonotonic. Crucially, the data shows a strong correspondence between $\eta$ and $S$, which confirms that for given $t$, the strength of the nonlinearity is the essential parameter which sets the postbuckling slope, and that a judicious choice of the metaparameters can lead to strongly discontinuous buckling.

Discontinuous buckling of slender beams.-Our scenario suggests that slender beams can exhibit discontinuous buckling when $\eta$ becomes sufficiently large. We have numerically determined the boundary in the $\ell-e$ plane between positive and negative $S$ for beams of thicknesses ranging from $9 \%$ to $1 \%$ [Fig. 5(a)]. As expected, to exhibit
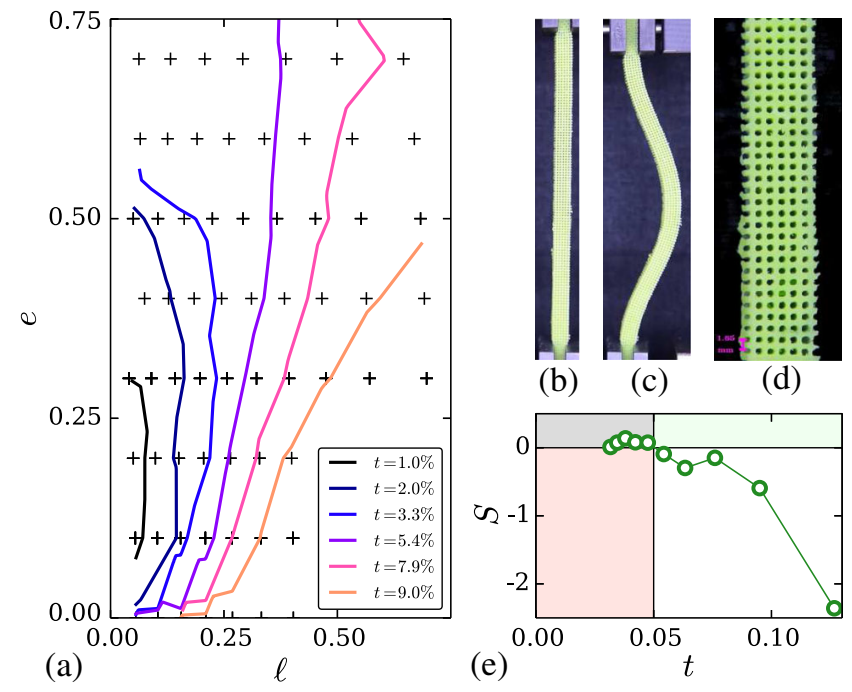

FIG. 5 (color online). Rational design of discontinuous buckling. (a) Boundaries between ordinary (right) and discontinuous (left) buckling in the $(e, \ell, t)$ parameter space. For each pair of $(e, \ell)$ values (crosses), simulations for a range of beam thicknesses have been performed. (b)-(d) Large metabeam $\left(L=520 \mathrm{~mm}, w=9.5 \mathrm{~mm}, d=16.0 \mathrm{~mm}, E=1.1 \times 10^{6} \mathrm{~Pa}\right)$ with 1980 holes with $e \approx 0.1$ and $\ell \approx 0.2$. (e) Experimental postbuckling slope $S$ vs aspect ratio $t$. discontinuous buckling, thinner beams require smaller values of $\ell$, tantamount to stronger nonlinearities. Pushing our computational power to the edge, we find numerical examples of $t=0.01$ beams that exhibit discontinuous buckling-here $\eta \approx 70$. Crucially, our data indicate that the critical value of $\ell$ scales linearly with $t$, so that suitable chosen metabeams can exhibit discontinuous buckling for arbitrary small values of the slenderness.

We also used this data to rationally design an experimental metabeam with desired postbuckling behavior. We pick a specific set of metaparameters $(e=0.1$, $\ell=0.2$ ) for which our numerics indicates that discontinuous buckling occurs for a critical aspect ratio $t \gtrsim 5 \%$ - see Fig. 5(a). We 3D print a mold consisting of $6 \times 330$ pillars (pitch $=1.65 \mathrm{~mm}$ ) with these parameters, and mold a beam of length $520 \mathrm{~mm}$, width $9.5 \mathrm{~mm}$ and 1980 holes-see Figs. 5(b)-5(d). By lateral clamping we vary the effective length $L_{e}$ of the metabeam, and thus its effective aspect ratio $t_{e}:=9.5 \mathrm{~mm} / L_{e}$ [31]. Figure 5(e) shows that discontinuous buckling sets in for $t_{e} \gtrsim 5 \%$, illustrating the success of our design strategy.

Discussion and outlook.-In this work, we showed how nonlinearity can alter the postbuckling mechanics of buckling: when the product of $|\eta|$ and critical strain is large enough, nonlinearities lead to discontinuous buckling. Whereas Euler theory is asymptotically valid for sufficiently linear materials when the thickness tends to zero, none of its current extensions $[28,29]$ actually predicts such qualitative change of the postinstability for realistic aspect ratios.

Our strategy is generic and opens up pathways for the rational design of other mechanical phenomena. We expect that this approach could be used to design the 2D buckling patterns [32] of metaplates. Could the snapping instability used in microactuators and sensors [33] be tweaked? Could we design metamaterials for which the postbuckling stiffness is larger than $1 / 2$ ? Finally, we note that most mechanical metamaterials have a beamlike microstructure [10,34-36], and often draw on buckling for their functionality [6-8,37-40]. We envision that tunable microscopic buckling will be of great use for the rational design of hierarchical metamaterials [36,41,42].

We thank J. Mesman for outstanding technical support. We acknowledge E.-J. Vegter, J. Lugthart, and R. Bastiaansen for exploratory experiments and theory, K. Kamrin, S. Neukirch, and S. Waitukaitis for discussions, NWO/VICI for funding, SEAS for hospitality.

[1] Y. Grabovsky and L. Truskinovsky, The flip side of buckling, Continuum Mech. Thermodyn. 19, 211 (2007).

[2] L. Euler, Additamentum I de curvis elasticis, methodus inveniendi lineas curvas maximi minimivi proprietate gaudentes, Opera Omnia, 1, 24, 245 (1744). 
[3] Z. Bazant and L. Cendolin, Stability of Structures (World Scientific, Singapore, 2009).

[4] We use large $d$ to avoid out of plane buckling.

[5] Polyvinyl Siloxane double elite 8, Young's modulus $E=250 \mathrm{kPa}$, Poisson's ratio $\nu \approx 0.5$.

[6] T. Mullin, S. Deschanel, K. Bertoldi, and M. C. Boyce, Pattern Transformation Triggered by Deformation, Phys. Rev. Lett. 99, 084301 (2007) .

[7] K. Bertoldi, P. M. Reis, S. Willshaw, and T. Mullin, Negative Poisson's ratio behavior induced by an elastic instability, Adv. Mater. 22, 361 (2010).

[8] K. Bertoldi, M. Boyce, S. Deschanel, S. Prange, and T. Mullin, Mechanics of deformation-triggered pattern transformations and superelastic behavior in periodic elastomeric structures, J. Mech. Phys. Solids 56, 2642 (2008).

[9] J. T. B. Overvelde, S. Shan, and K. Bertoldi, Compaction through buckling in 2D periodic, soft and porous structures: Effect of pore shape, Adv. Mater. 24, 2337 (2012).

[10] B. Florijn, C. Coulais, and M. van Hecke, Programmable Mechanical Metamaterials, Phys. Rev. Lett. 113, 175503 (2014).

[11] M. Taylor, L. Francesconi, M. Gerendás, A. Shanian, C. Carson, and K. Bertoldi, Low porosity metallic periodic structures with negative Poisson's ratio, Adv. Mater. 26, 2365 (2014).

[12] Polyvinyl Siloxane double elite 32, Young's modulus $E=1.1 \mathrm{MPa}$, Poisson's ratio $\nu \approx 0.5$.

[13] The range over which we can measure the postbuckling slope is limited, as at larger values of $\tilde{\varepsilon}$ the experimental beams develop a crease or sulcus [14-17], which is outside the scope of this work.

[14] A. N. Gent, Elastic instabilities in rubber, Int. J. Nonlinear Mech. 40, 165 (2005).

[15] A. Ghatak and A. L. Das, Kink Instability of a Highly Deformable Elastic Cylinder, Phys. Rev. Lett. 99, 076101 (2007)

[16] E. Hohlfeld and L. Mahadevan, Unfolding the Sulcus, Phys. Rev. Lett. 106, 105702 (2011).

[17] E. Hohlfeld and L. Mahadevan, Scale and Nature of Sulcification Patterns, Phys. Rev. Lett. 109, 025701 (2012).

[18] J. W. Hutchinson and W. T. Koiter, Postbuckling theory, Appl. Mech. Rev. 23, 1353 (1970).

[19] L. Pocivavsek, R. Dellsy, A. Kern, S. Johnson, B. Lin, K. Y. C. Lee, and E. Cerda, Stress and fold localization in thin elastic membranes, Science 320, 912 (2008).

[20] H. Diamant and T. A. Witten, Compression Induced Folding of a Sheet: An Integrable System, Phys. Rev. Lett. 107, 164302 (2011).

[21] B. Audoly, Localized buckling of a floating elastica, Phys. Rev. E 84, 011605 (2011).

[22] M. C. Boyce and E. M. Arruda, Constitutive models of rubber elasticity: A review, Rubber Chem. Technol. 73, 504 (2000).

[23] R. W. Ogden, Non Linear Elasic Deformations (Dover, New York, 1997).
[24] See Supplemental Material at http://link.aps.org/ supplemental/10.1103/PhysRevLett.115.044301 for details about the numerical scheme.

[25] The 2D plane strain approximation describes our 3D experimental situations, where the depth of the beam is much larger than its width.

[26] Clearly this expansion cannot be extended down to $\tilde{\varepsilon}=0$. See Supplemental Material at http://link.aps.org/ supplemental/10.1103/PhysRevLett.115.044301 for the details about the calculation of $\eta$ in the simple case of uniaxial compression.

[27] L. A. Lubbers, C. Coulais, and M. van Hecke, Elastica for Nonlinear Beams (to be published).

[28] A. Magnusson, M. Ristinmaa, and C. Ljung, Behaviour of the extensible elastica solution, Int. J. Solids Struct. 38, 8441 (2001).

[29] A. Humer, Exact solutions for the buckling and postbuckling of shear-deformable beams, Acta Mech. 224, 1493 (2013).

[30] See the Supplemental Material at http://link.aps.org/ supplemental/10.1103/PhysRevLett.115.044301 for movies.

[31] Such clamping boundary conditions are not exactly the same as the no-slip boundary conditions used before.

[32] B. Audoly and Y. Pomeau, Elasticity and Geometry (Oxford University, New York, 2010).

[33] M. P. Brenner, J. H. Lang, J. Li, J. Qiu, and A. H. Slocum, Optimal design of a bistable switch, Proc. Natl. Acad. Sci. U.S.A. 100, 9663 (2003).

[34] M. Kadic, T. Bückmann, N. Stenger, M. Thiel, and M. Wegener, On the practicability of pentamode mechanical metamaterials, Appl. Phys. Lett. 100, 191901 (2012).

[35] M. Kadic, T. Bückmann, R. Schittny, and M. Wegener, Metamaterials beyond electromagnetism, Rep. Prog. Phys. 76, 126501 (2013).

[36] R. Oftadeh, B. Haghpanah, D. Vella, A. Boudaoud, and A. Vaziri, Optimal Fractal-Like Hierarchical Honeycombs, Phys. Rev. Lett. 113, 104301 (2014).

[37] P. Wang, F. Casadei, S. Shan, J. C. Weaver, and K. Bertoldi, Harnessing Buckling to Design Tunable Locally Resonant Acoustic Metamaterials, Phys. Rev. Lett. 113, 014301 (2014).

[38] J. T. B. Overvelde and K. Bertoldi, Relating pore shape to the nonlinear response of periodic elastomeric structures, J. Mech. Phys. Solids 64, 351 (2014).

[39] J. Shim, C. Perdigou, E. R. Chen, K. Bertoldi, and P. M. Reis, Buckling-induced encapsulation of structured elastic shells under pressure, Proc. Natl. Acad. Sci. U.S.A. 109, 5978 (2012).

[40] S. Babaee, J. Shim, J. C. Weaver, N. Patel, and K. Bertoldi, 3D soft metamaterials with negative Poisson's ratio, Adv. Mater. 25, 5044 (2013).

[41] Y. Cho et al., Engineering the shape and structure of materials by fractal cut, Proc. Natl. Acad. Sci. U.S.A. 111, 17390 (2014).

[42] R. Lakes, Materials with structural hierarchy, Nature (London) 361, 511 (1993). 


\title{
Supplemental Information for the paper: Discontinuous Buckling of Wide Beams and Metabeams, I
}

\author{
Corentin Coulais, ${ }^{1,2}$ Johannes T. B. Overvelde, ${ }^{3}$ Luuk A. Lubbers, ${ }^{1,2}$ Katia Bertoldi, ${ }^{3}$ and Martin van Hecke ${ }^{1,2}$ \\ ${ }^{1}$ Huygens-Kamerlingh Onnes Lab, Universiteit Leiden, PObox 9504, 2300 RA Leiden, The Netherlands \\ ${ }^{2}$ FOM Institute AMOLF, Science Park 104, 1098 XG Amsterdam, The Netherlands \\ ${ }^{3}$ School of Engineering and Applied Sciences, Harvard university, Cambridge, Massachusetts 02138, USA
}

\section{Numerical Method}

We perform implicit finite element simulations with the commercial software Abaqus. For the 3D simulations (see fig. 2 of the main text), we use hybrid quadratic brick elements (Abaqus type C3D20H). For the 2D simulations, (see figs. 3, 4 and 5 of the main text), we use 2D quadratic plane strain elements (Abaqus type CPE8). For the plain beams, the elements are quadrilateral and for the metabeams, they are triangular. Metabeams with an even number of holes across their thickness are asymmetric. Therefore, in order to run robust simulations with fully symmetric initial geometries, we only consider metabeams with an odd number of holes across their width. To capture the instability without seeding the material with imperfections, we develop a two-steps protocol. First we perform a nonlinear stability analysis to determine the bifurcation point $\left(u_{b}, F_{b}\right)$ with a relative accuracy of $10^{-3}$. Second, to probe the post buckling branch for $F>F_{b}$, we apply a transverse perturbation at the center of the beam and release it once the beam is in the bifurcated - buckled - state. We then probe the buckled branch by smoothly increasing the compression, $u$, to $3 u_{b}$. This protocol allows to determine the location of the instability and the post-buckling behavior with high accuracy.

\section{Nonlinear Stiffening of Hyper-Elastic Beams.}

We consider a rubber beam under uniaxial loading in the direction $z$ and no deformations in the $y$ direction -Fig. S1. The strain energy density for incompressible neo-Hookean materials is [1]

$$
W=\frac{E}{6}\left(\lambda_{x}^{2}+\lambda_{y}^{2}+\lambda_{z}^{2}-3\right),
$$

where $\lambda_{i}$, are the stretches in the principal directions $i$ (length ratio before/after deformation), $E$ is the Young's modulus. The incompressibility assumption translates into the equation $\lambda_{x} \lambda_{y} \lambda_{z}=1$. For homogeneous deformations under constraints, the equilibrium state of the sample is simply given by

$$
\sigma_{i}=\lambda_{i} \frac{\partial W}{\partial \lambda_{i}}-\Pi
$$

where $\sigma_{i}$ is the Cauchy stress and $\Pi$ is a Lagrange Multiplier [1]. Since the deformations are spatially homogeneous, the $\lambda_{i}$ are constant in the sample and $\lambda_{z}$ relates then simply to the vertical nominal strain $\tilde{\varepsilon}_{z z}$, by $\lambda_{z}=1+\tilde{\varepsilon}_{z z}$.

Here, we consider a uniaxial compression in the $z$ direction of a beam with no stresses in the $x$ directions and no deformations in the $y$ direction, therefore, the transverse stress $\sigma_{x}=0$ and the transverse stretch $\lambda_{y}=1$. We thus obtain

$$
\Pi=\lambda_{x} \frac{\partial W}{\partial \lambda_{x}}
$$

Therefore, the third equilibrium equation gives

$$
\begin{aligned}
\sigma_{z} & =\lambda_{z} \frac{\partial W}{\partial \lambda_{z}}-\lambda_{x} \frac{\partial W}{\partial \lambda_{x}} \\
& =\frac{E}{3}\left(\lambda_{z}^{2}-\lambda_{x}^{-2}\right) .
\end{aligned}
$$

Using the incompressibility condition and the hypothesis $\lambda_{y}=1$, we obtain $\lambda_{x}=\lambda_{z}^{-1}$, so that

$$
\sigma_{z}=\frac{E}{3}\left(\lambda_{z}^{2}-\frac{1}{\lambda_{z}^{2}}\right)
$$


(a)

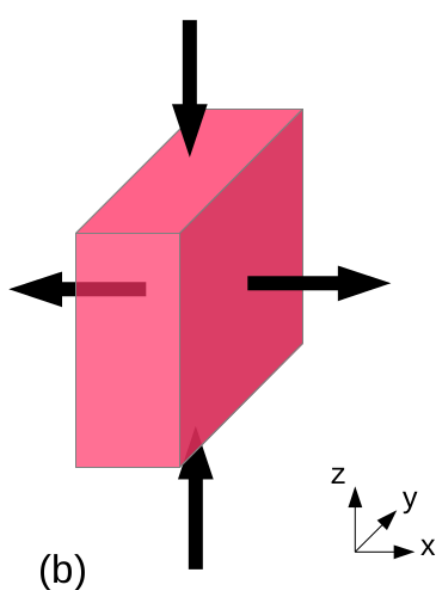

FIG. S1: Beam under uniaxial compression in the undeformed (left) and deformed (right) states with no deformation in the $y$ direction (also called plane strain conditions). The deformation are homogeneous and boundary conditions impose $\sigma_{x}=0$ and $\lambda_{y}=0$.

Finally, since the Cauchy stress and the stretch relate to the nominal stress as $\tilde{\sigma}_{z z}=\sigma_{z} / \lambda_{z}[1]$, we can write

$$
\tilde{\sigma}_{z z}=\frac{E}{3}\left(\lambda_{z}-\frac{1}{\lambda_{z}^{2}}\right)=\frac{E}{3}\left(1+\tilde{\varepsilon}_{z z}-\frac{1}{\left(1+\tilde{\varepsilon}_{z z}\right)^{3}}\right) .
$$

Therefore,

$$
\frac{\tilde{\sigma}_{z z}}{E}=\frac{1}{3}\left(1+\tilde{\varepsilon}_{z z}-\left(1+\tilde{\varepsilon}_{z z}\right)^{-3}\right) .
$$

This result is a good approximation for the pre-buckling stage of our 3D beams, although in the experiment, the hypothesis $\lambda_{y}=1$ is not strictly true.

[1] R. W. Ogden, Non Linear Elasic Deformations (Dover Publ, 1997). 